\title{
Higher incidence of morbidity in women than men with non-functioning pituitary adenoma: a Swedish nationwide study
}

\author{
Daniel S Olsson', Ing-Liss Bryngelsson² and Oskar Ragnarsson' \\ ${ }^{1}$ Department of Endocrinology, Institute of Medicine, Sahlgrenska Academy, University of Gothenburg \\ and Sahlgrenska University Hospital, Gothenburg, Sweden and ${ }^{2}$ Department of Occupational and \\ Environmental Medicine, Örebro University Hospital, Örebro, Sweden
}

\author{
Correspondence \\ should be addressed \\ to D S Olsson \\ Email \\ daniel.olsson@gu.se
}

\begin{abstract}
Objective: Increased mortality rates are found in women and young adults with non-functioning pituitary adenomas (NFPAs). This nationwide study aimed to investigate the burden of comorbidities in patients with NFPA and to examine whether gender influences the outcome.

Design: NFPA patients were identified and followed-up from National Registries in Sweden. It was a nationwide, population-based study.

Method: Standardised incidence ratios (SIRs) for comorbidities with $95 \%$ confidence intervals (CI). Comorbidities were analysed in all patients, both patients with and without hypopituitarism.

Results: Included in the analysis were 2795 patients (1502 men, 1293 women), diagnosed with NFPA between 1987 and 2011. Hypopituitarism was reported in 1500 patients (54\%). Mean patient-years at risk per patient was 7 (range 0-25). Both men (SIR 2.2, 95\% CI: 1.8-2.5; $P<0.001)$ and women $(2.9,2.4-3.6 ; P<0.001)$ had a higher incidence of type 2 diabetes mellitus (T2DM) than the general population, with women having a higher incidence compared with men $(P=0.02)$. The incidence of myocardial infarction was increased in women $(1.7,1.3-2.1 ; P<0.001)$, but not in men.

Both men $(1.3,1.1-1.6 ; P=0.006)$ and women $(2.3 ; 1.9-2.8 ; P<0.001)$ had an increased incidence of cerebral infarction, with women having a higher incidence than men $(P<0.001)$. The incidence of sepsis was increased for both genders. The incidence of fractures was increased in women $(1.8,1.5-1.8 ; P<0.001)$, but not for men. Conclusions: This nationwide study shows excessive morbidity due to T2DM, cerebral infarction and sepsis in all NFPA patients. Women had higher incidence of T2DM, myocardial infarction, cerebral infarction and fracture in comparison to both the general population and to men.

\section{Introduction}

Patients with hypopituitarism have an increased mortality ratio, mainly due to respiratory, cardiovascular and cerebrovascular causes $(1,2,3,4,5)$. The reason for the excessive mortality is currently not fully understood although some factors associated with increased risk have been identified. Patients diagnosed at young age have 2.7 times higher mortality compared with the general population (6). Women have also an increased risk of premature death compared with men as well as (c) 2016 European Society of Endocrinology Printed in Great Britain patients with pituitary adenoma operated by transcranial surgery compared with those treated by transphenoidal surgery (3). Pituitary radiation therapy, untreated growth hormone deficiency and high glucocorticoid replacement doses may also implicate increased risk $(2,3,7,8,9)$.

As almost all previous studies have investigated heterogeneous groups of patients with hypopituitarism of various aetiologies, the increased mortality may not solely be due to the hormone deficiencies but also to the

Published by Bioscientifica Ltd. 
underlying disease itself, such as Cushing's disease or craniopharyngioma. In an attempt to bypass this potential bias, we have recently studied mortality in a large cohort of patients with a single aetiology, non-functioning pituitary adenomas (NFPAs) (6). Despite this approach, with a homogenous population, an increased mortality was observed, especially among women and patients diagnosed with NFPA before the age of 40 years. To date, there is no explanation for the worse prognosis in women and young patients.

The aim of this nationwide population study was to investigate the burden of cerebral infarctions, myocardial infarctions, type 2 diabetes mellitus, fractures and sepsis in patients with NFPA and to examine whether gender and age at diagnosis influence the outcome.

\section{Subjects and methods}

\section{Study design}

Patients with NFPA diagnosis were identified in The Swedish National Patient Registry (Patient Registry). The registry achieved national coverage in 1987 and contains information from every patient visit within the Swedish hospital system. An individual patient can be followed throughout life in the Swedish National Health registries using a unique Swedish personal identification number. The National Board of Health and Welfare secures high quality for the Patient Registry (10). To identify patients with NFPA diagnosis, we used a combination of identification criteria to ensure a high quality in the selection of the patients. The identification process has been previously described in detail in a study when mortality in the Swedish national cohort of patients with NFPA was studied (8). In short, patients should have been diagnosed with NFPA (International Classification of Diseases, 10th revision (ICD-10) code D35.2) either at a medical/endocrine care unit or at a neurological or neurosurgical care unit. This method of combined criteria for identification (both diagnostic codes and department codes) has previously been shown to achieve a high sensitivity and specificity when selecting patients with pituitary diseases in epidemiological research $(11,12)$. All patients with a diagnosis of a GH-, ACTH-, or TSHsecreting adenoma; prolactinoma; craniopharyngioma; pituitary cyst or a pinealoma were excluded before the inclusion criteria were applied to the registries. To further ensure a high quality, an internal validation of the selection criteria was performed by reviewing the NFPA diagnosis of patients originating from the Sahlgrenska
University Hospital's uptake area (17\% of the total patient population) against patient records. The NFPA diagnosis was confirmed in 424 patients, which resulted in a positive predictive value of $91 \%$. Other non-functioning pituitary tumours, mostly cystic pituitary tumours, were found in $4 \%$ of the patients. Only $1 \%$ of the patients had a lesion not affecting the sella region (6). In patients with cystic pituitary tumours, 11 out of 14 patients were women.

Patients with NFPA were identified in the Patient Registry from 1 January 1997 (when ICD-10 classification was implemented in Sweden) to 31 December 2011. The date of the NFPA diagnosis was also gathered from the Patient Registry. Patients that had deceased or emigrated before 1 January 1997 were not included in the cohort. The time at risk for the patients with NFPA started on the date of diagnosis (date of the first registered NFPA diagnosis). The start of the investigation period was 1 January 1987. The time at risk, in which patients were studied regarding morbidity, ended on the date of death or at the end of the study, 31 December 2011. Date of birth, gender, diagnoses of hypopituitarism and diabetes insipidus (DI) and radiotherapy were collected from the Patient Registry. The degree of hypopituitarism and its management were not available in the registry. Information regarding radiotherapy was obtained in patients diagnosed between 1 January 1997 and 31 December 2011 (during the ICD-10 classification period). Morbidity data for cerebral infarctions, myocardial infarctions, diabetes mellitus, fractures and sepsis were also collected from the Patient Registry and classified in accordance with the ICD classification (for ICD-codes, Supplementary Material, see section on supplementary data given at the end of this article). Data on comorbidities in the general population were gathered and analysed in the same manner as for the patients.

\section{Ethics}

The study was approved by the Regional Ethical Review Board in Gothenburg, Sweden, and by the National Board of Health and Welfare, Sweden.

\section{Statistical analysis}

Person-years at risk were calculated from study inclusion to death or end of study and stratified according to gender, 5-year age groups and 1-year calendar periods. The expected number of cases for each stratum was calculated using the general Swedish population as reference. The observed number of comorbidities among subjects with 
NFPA was compared with those expected in the general population using standardised incidence ratios (SIRs). SIRs for each diagnosis were calculated depending on whether the patients received the specific diagnosis on a yearly basis with the exception of type 2 diabetes mellitus, which was analysed based on the first event only. Ninetyfive percent confidence intervals (CIs) were calculated assuming a Poisson distribution of the observed numbers. Subgroup analyses for gender, age at diagnosis, hormonal deficiencies and radiotherapy were performed. If fewer than four events were observed, no estimate was made for SIR. SIRs for non-overlapping subgroups were compared with each other (13). No patients in the validated subgroup originating from the Sahlgrenska University Hospital's uptake area were lost to follow-up, e.g. due to emigration. IBM SPSS (version 21; SPSS) and STATA/SE (version 12.1; Statacorp) software were used for the statistical analyses.

\section{Results}

This nationwide study included 2795 unique patients with NFPA (1502 men and 1293 women; Table 1). The mean age at diagnosis was $58.4 \pm 17$ years and the mean personyears at risk per patient was 7.2 (range $0-25$ ) years. The length of the person-years at risk per patient did not differ between men ( 7.2 years \pm 5.4 ) and women ( 7.2 years \pm 5.2 ). In total, 20139 patient-years were included in the study.

Table 1 Characteristics of patients with non-functioning pituitary adenoma.

\begin{tabular}{l}
\hline \\
\hline Gender, $n$ (\%) \\
Men \\
Women \\
Age at diagnosis, y, mean (range) \\
Men, mean \pm s.D. \\
Women, mean \pm s.D. \\
Mean patient-years at risk per patient, \\
y (range) \\
Patient-years at risk in the study \\
Hormonal status, $n$ (\%) \\
Patients with hypopituitarism \\
Patients with diabetes insipidus \\
Patients without any hormonal deficiencies \\
Patients diagnosed at or before 40 years of \\
age, $n$ (\%) \\
Time of diagnosis, $n$ (\%) \\
Patients diagnosed before 1 January 1997 \\
Patients diagnosed between 1 January \\
1997 and 31 December 2011 \\
Radiotherapy of patients diagnosed \\
between 1 January 1997 and \\
31 December 2011
\end{tabular}

\begin{tabular}{c}
\hline Total $(n=2795)$ \\
\hline $1502(54)$ \\
$1293(46)$ \\
$58.4(1-97)$ \\
$60.2 \pm 15$ \\
$56.2 \pm 18$ \\
$7(0-25)$ \\
20139 \\
$1500(54)$ \\
$145(5)$ \\
$1266(45)$ \\
$423(15)$ \\
\\
$322(12)$ \\
$2473(88)$ \\
$104(4)$
\end{tabular}

The diagnoses of hypopituitarism and DI were recorded in 1500 patients $(54 \%)$ and in 145 patients (5\%) respectively. More men $(n=957,63 \%)$ than women $(n=572,44 \%)$ were diagnosed with hypopituitarism and/or diabetes insipidus $(P<0.0001)$. A total of 104 patients $(4 \%)$ had received treatment with radiotherapy.

\section{Overall morbidity}

In the whole cohort of NFPA patients, the incidence of type 2 diabetes mellitus and sepsis was more than doubled compared with the general population (Fig. 1 and Table 2). The incidence of cerebral infarction was increased by over $60 \%$, whereas the incidence of myocardial infarction was only slightly elevated compared with that expected. The incidence of fracture was increased by over $40 \%$ for the patients.

In the subgroup of patients treated $(n=104)$ with radiation therapy, seven patients had a cerebral infarction compared with the expected number of $3.5(P=0.14)$.

\section{Men and women}

The incidences (SIR, 95\% CI) of type 2 diabetes mellitus (women $2.9,2.4-3.6$ vs men $2.2,1.8-2.5 ; P=0.016$ ) and cerebral infarction (women 2.3, 1.9-2.8 vs men 1.3, 1.1-1.6; $P<0.0001$ ) were significantly higher in women than in men (Table 2). Furthermore, the incidences of myocardial infarction and fracture were increased for women, whereas they were as expected for men. There

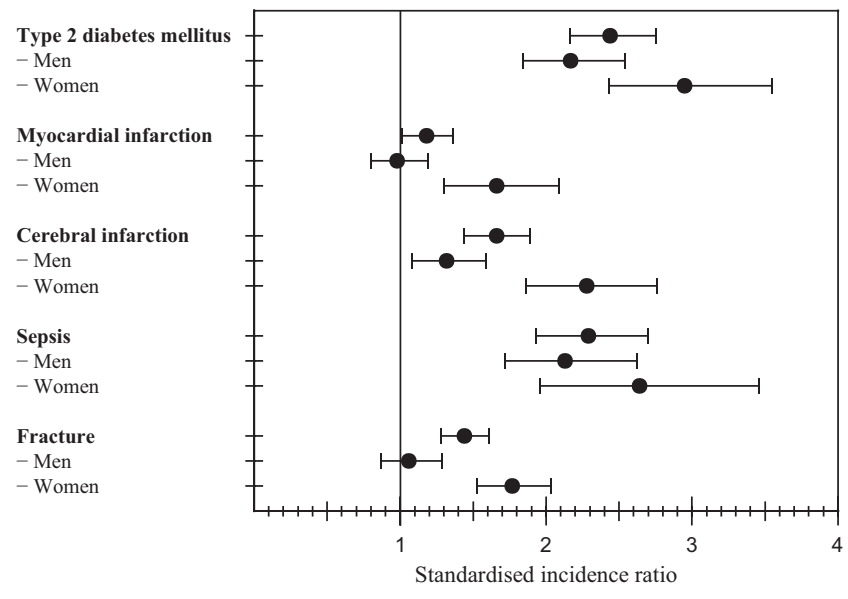

Figure 1

Morbidity in non-functioning pituitary adenomas presented as standardised incidence ratios and $95 \% \mathrm{Cls}$ in the whole cohort and sub-grouped according to gender. 
Table 2 Standardised incidence ratios for patients with non-functioning pituitary adenoma.

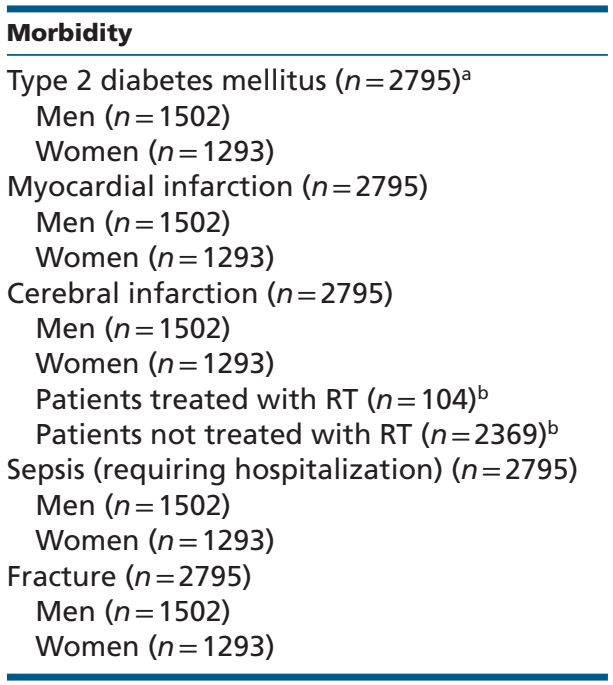

\begin{tabular}{ccc} 
No. expected & No. diagnosed \\
\cline { 1 - 1 } 109.8 & & 268 \\
71.8 & & 156 \\
38.0 & & 112 \\
150.2 & & 177 \\
106.9 & & 105 \\
43.3 & & 72 \\
129.2 & & 214 \\
83.5 & & 110 \\
45.7 & & 104 \\
3.5 & & 7 \\
91.4 & & 135 \\
62.5 & & 143 \\
43.1 & & 92 \\
19.4 & 51 \\
211.6 & & 305 \\
98.7 & 105 \\
113.0 & & 200 \\
\hline
\end{tabular}

\begin{tabular}{ccc}
\hline SIR $(95 \% \mathrm{CI})$ & & P-value \\
$2.44(2.16-2.75)$ & & $<0.0001$ \\
$2.17(1.84-2.54)$ & $<0.0001$ \\
$2.95(2.43-3.55)$ & $<0.0001$ \\
$1.18(1.01-1.36)$ & 0.036 \\
$0.98(0.80-1.19)$ & 0.91 \\
$1.66(1.30-2.09)$ & $<0.0001$ \\
$1.66(1.44-1.89)$ & $<0.0001$ \\
$1.32(1.08-1.59)$ & 0.006 \\
$2.28(1.86-2.76)$ & $<0.0001$ \\
$1.98(0.79-4.07)$ & 0.14 \\
$1.48(1.24-1.75)$ & $<0.0001$ \\
$2.29(1.93-2.70)$ & $<0.0001$ \\
$2.13(1.72-2.62)$ & $<0.0001$ \\
$2.64(1.96-3.46)$ & $<0.0001$ \\
$1.44(1.28-1.61)$ & $<0.0001$ \\
$1.06(0.87-1.29)$ & 0.55 \\
$1.77(1.53-2.03)$ & $<0.0001$ \\
\hline
\end{tabular}

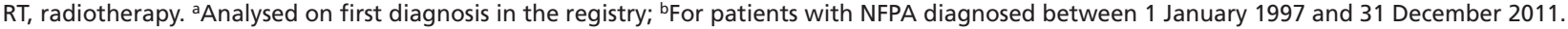

was no significant difference for the incidence of sepsis requiring hospitalization between the genders (women $2.6,2.0-3.5$ vs men $2.1,1.7-2.6 ; P=0.24)$.

Women with hypopituitarism and/or DI had an increased incidence of myocardial infarction, which was not seen for women without hormonal deficiencies (Table 3). Also, the incidence of cerebral infarctions was higher in women with hormonal deficiencies compared with non-deficient women (SIR 2.8, 95\% CI: $2.2-3.6$ vs $1.7,1.2-2.3 ; P=0.015)$. There was no statistically significant difference in the incidence of sepsis between women with and without hormonal deficiencies $(3.3,2.3-4.6$ vs $1.9,1.1-3.0 ; P=0.077)$. Men without hormonal deficiencies had a worse outcome with respect to type 2 diabetes mellitus, myocardial infarction and fracture compared with men with hypopituitarism and/or DI.

\section{Young age at diagnosis}

For patients who were diagnosed with NFPA before or at 40 years of age the incidences of type 2 diabetes mellitus, cerebral infarction and sepsis requiring hospitalization were clearly increased compared with the general population (Table 4). Women diagnosed at a young age had an increased incidence of type 2 diabetes mellitus, which was not seen for men.

\section{Discussion}

This nationwide study demonstrated an increased incidence of type 2 diabetes mellitus, myocardial infarction, cerebral infarction, sepsis and fractures in patients with NFPA compared with the general population. The study, the largest one of patients with NFPA to date, provides a new insight into the condition and stresses the importance of early detection and adequate preventive management of comorbidities in patients with NFPA.

Women with pituitary insufficiency have a higher mortality ratio than men $(1,2,3,5,14)$. In accordance with previous studies on mortality, women with NFPA seem to be especially vulnerable for comorbidities. In fact, women in our cohort had excess incidences of myocardial infarction and fractures, whereas men did not. Also, the increased incidences of diabetes mellitus and cerebral infarction that were observed in both genders were significantly higher in women than in men. The reason for these gender differences is unclear although results from previous studies indicate that relatively higher glucocorticoid doses in women (glucocorticoid dose per kg body weight), untreated growth hormone deficiency and the quality of sex steroid replacement may be influencing factors $(2,15,16)$. It has also been suggested that the natural survival advantage that women have over men disappears with pituitary insufficiency (5) and that hypopituitarism is more often underdiagnosed in women compared with men (17). In the current study, more men than women were diagnosed with pituitary insufficiency. Thus, underdiagnosis, and thereof undertreatment, may contribute to the differences observed between the genders.

The cohort in the current study included 423 patients diagnosed with NFPA at or before the age of 40 years. In accordance with previous epidemiological studies on 
Table 3 Morbidity in patients with non-functioning pituitary adenoma with and without hormonal deficiencies.

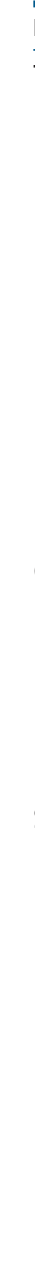

Morbidity

Type 2 diabetes mellitus ${ }^{a}$

Men

With hypopituitarism and/or DI $(n=957)$

Without hypopituitarism and/or DI $(n=545)$

Women

With hypopituitarism and/or DI $(n=572)$

Without hypopituitarism and/or DI $(n=721)$

Myocardial infarction

Men

With hypopituitarism and/or DI $(n=957)$

Without hypopituitarism and/or DI $(n=545)$

Women

With hypopituitarism and/or DI $(n=572)$

Without hypopituitarism and/or DI $(n=721)$

Cerebral infarction

Men

With hypopituitarism and/or DI $(n=957)$

Without hypopituitarism and/or DI $(n=545)$

Women

With hypopituitarism and/or DI $(n=572)$

Without hypopituitarism and/or DI $(n=721)$

Sepsis (requiring hospitalization)

Men

With hypopituitarism and/or DI $(n=957)$

Without hypopituitarism and/or DI $(n=545)$

Women

With hypopituitarism and/or DI $(n=572)$

Without hypopituitarism and/or DI $(n=721)$

Fracture

Men

With hypopituitarism and/or DI ( $n=957)$

Without hypopituitarism and/or DI $(n=545)$

Women

With hypopituitarism and/or DI $(n=572)$

Without hypopituitarism and/or DI $(n=721)$
No. expected

No. diagnosed

SIR $(95 \% \mathrm{Cl})$

51.9

19.9

20.7

17.3

94

62

66

46

$1.81(1.46-2.22)$

$3.11(2.38-3.99)$

$3.19(2.47-4.06)$

2.66 (1.95-3.55)

$0.82(0.63-1.05)$

$P$-value

77.1

29.8

23.3

20.0

45

59.9

23.6

75

24.6

21.1

30.5

12.6

65

10.3

34

9.0

69.7

29.0

59.7

53.3
$1.41(1.01-1.90)$

$1.93(1.41-2.58)$

1.35 (0.89-1.96)

27

35

69

35

27

63

2

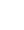

$.25(0.98-1.57)$

$1.48(1.03-2.06)$

$2.80(2.18-3.55)$

$1.66(1.16-2.31)$

$2.14(1.41-3.12)$

3.29 (2.28-4.59)

1.89 (1.10-3.02)

$0.88(0.67-1.12)$

$1.52(1.10-2.04)$

$1.73(1.41-2.09)$

$1.82(1.48-2.22)$
$2.13(1.64-2.72)$
$<0.0001$

$<0.0001$

$<0.0001$

$<0.0001$

0.12

0.041

$<0.0001$

0.16

0.066

0.034

$<0.0001$

0.0068

$<0.0001$

0.0004

$<0.0001$

0.017

0.29

0.0090

$<0.0001$

$<0.0001$

DI, diabetes insipidus. ${ }^{a}$ Analysed on first diagnosis in the registry.

Table 4 Morbidity in 423 young adult (age $\leq 40$ years) patients with non-functioning pituitary adenoma.

\begin{tabular}{l}
\hline Morbidity \\
\hline Type 2 diabetes mellitus \\
Young adult patients \\
Men $(n=173)$ \\
Women $(n=250)$ \\
Myocardial infarction \\
Young adult patients \\
Cerebral infarction \\
Young adult patients \\
Sepsis (requiring hospitalization) \\
Young adult patients \\
Fracture \\
Young adult patients \\
Men $(n=173)$ \\
$\quad$ Women $(n=250)$ \\
\hline
\end{tabular}

\begin{tabular}{c}
\hline No. expected \\
\hline 1.9 \\
1.1 \\
0.8 \\
1.3 \\
0.9 \\
1.4 \\
\\
\\
\\
\\
5.8 \\
3.7 \\
\hline
\end{tabular}

\begin{tabular}{c}
\hline No. diagnosed \\
\hline 9 \\
4 \\
5 \\
1 \\
5 \\
5 \\
6 \\
1 \\
5
\end{tabular}

\begin{tabular}{|c|}
\hline SIR $(95 \% \mathrm{CI})$ \\
\hline $\begin{array}{l}4.62(2.11-8.77) \\
3.58(0.98-9.16) \\
6.02(1.95-14.1)\end{array}$ \\
\hline NC \\
\hline 5.75 (1.87-13.4) \\
\hline $3.65(1.18-8.51)$ \\
\hline $\begin{array}{c}0.68(0.25-1.48) \\
N C \\
1.37(0.44-3.19)\end{array}$ \\
\hline
\end{tabular}

\begin{tabular}{c}
$P$-value \\
0.0004 \\
0.054 \\
0.0033 \\
NC \\
0.0040 \\
0.026 \\
0.44 \\
NC \\
0.61 \\
\hline
\end{tabular}

NC, not calculated. aAnalysed on first diagnosis in the registry. If the number of events was five or less, figures were not divided depending on gender. 
mortality in patients with hypopituitarism $(3,6)$, young patients with NFPAs were shown to be especially exposed with 3 to 6 times increased incidences of type 2 diabetes mellitus, cerebral infarction and sepsis. Even though the number of individual cases with these comorbidities was relatively small, and the $95 \%$ CIs were consequently wide, preventive measures for patients diagnosed with NFPAs at young age should not be underestimated.

Hypopituitary patients have excess vascular mortality and increased occurrence of the metabolic syndrome, diabetes mellitus, cardiac diseases and non-fatal stroke (1, $2,3,16,18,19)$. The reason for this is probably multifactorial, with non-physiologic glucocorticoid replacement, untreated growth hormone deficiency and undertreatment with sex steroids being suggested explanations (2, 20). Our results, showing higher incidences of type 2 diabetes mellitus, myocardial infarction and cerebral infarction among patients with NFPAs, are in line with previous studies. Pituitary function only seemed to influence the outcome in women, but not in men. In fact, the incidence ratios in women for myocardial and cerebral infarctions were negatively affected by pituitary insufficiencies, whereas the incidences of type 2 diabetes mellitus were not affected. Furthermore, men without hypopituitarism had higher incidences of type 2 diabetes mellitus than men with pituitary insufficiency. Other factors, yet unidentified, must, therefore, be involved in the development of adverse outcome in patient with NFPAs. Further studies are needed to illuminate which factors influence cardiovascular outcome in patients with NFPA, not only the influence of pituitary hormone replacement but also the impact of factors such as socioeconomic status, physical activity, dietary habits and cognitive function.

An interesting finding in this study was the higher incidence of sepsis requiring hospitalization in both men and women with NFPA. Awareness of this increased risk is of great importance, especially for patients with secondary adrenal insufficiency. In fact, previous study has shown a significant increased mortality in hypopituitary patients due to infectious diseases and that all of the patients who died were ACTH insufficient (21). In our study, the incidence of sepsis was not different between patients with pituitary insufficiency and those with normal pituitary function. Although not statistically significant, sepsis tended to be more common in hypopituitary women compared with female patients with intact pituitary function $(P=0.077)$.

Patients with pituitary insufficiency have decreased bone mineral density and increased fracture risk $(22,23$, 24). In the current study, we demonstrate that women with NFPA have an almost two-fold increased incidence of fractures compared with the background population, while men did not have an increased risk. Interestingly, no difference in incidence of fracture was seen when women with and without pituitary insufficiency were compared with each other.

The main strength of the study is the large number of patients included, with more than 20000 patient-years of follow-up, which makes the study the largest so far on patients with NFPA. Also, the identification process with multiple inclusion and exclusion criteria as well as an internal validation of the NFPA diagnosis in $17 \%$ of the patients showing that over $90 \%$ had a correct diagnosis, which is a high proportion, adds to the strengths of the study. However, there were also some limitations. Due to the nature of the study, based on registry data, detailed information on tumour treatment and the degree of hypopituitarism and its management were not available in the registry. Thus, the role of glucocorticoid, growth hormone, sex hormone and L-thyroxin replacement therapy on the comorbidities could not be analysed and the results concerning hormonal status should, therefore, be interpreted with caution. However, the validity of the variable "pituitary insufficiency" has been investigated previously in patients with craniopharyngioma with reassuring results (12).

In conclusion, this nationwide study demonstrates an excessive morbidity due to type 2 diabetes mellitus, myocardial infarction, cerebral infarction, sepsis and fracture in patients with NFPA. Also, women and young adults with NFPA seem to be especially vulnerable to develop comorbidities. The results emphasize the importance of an active surveillance in all patients diagnosed with NFPA as well as adequate preventive treatments in high-risk patients.

Supplementary data

This is linked to the online version of the paper at http://dx.doi.org/10.1530/ EJE-16-0173.

Declaration of interest

D S O has been a consultant for Novartis, Ipsen and Pfizer. I L B and O R have nothing to disclose.

Funding

The Sahlgrenska University Hospital Fund and The Swedish government under the ALF-agreement.

Author contributions statement

All authors took part in the design of the study. D S O and I L B performed the patient selection process and data collection. D SO designed the figure. All authors contributed to data interpretation and analysis, and in writing and revising the report. All authors are responsible for the integrity of the data and accuracy of the analysis, and all approved the final report. 


\section{Acknowledgment}

We would like to express our gratitude to the staff at the Centre for Endocrinology and Metabolism at the Department of Endocrinology at Sahlgrenska University Hospital and to The National Board of Health and Welfare for their excellent collaboration. We would also like to thank Professor Bengt-Åke Bengtsson for his valuable comments on the manuscript. We thank Peter Todd (Tajut Ltd, Kaiapoi, New Zealand) for third-party writing assistance in the drafting of this manuscript, for which he received financial compensation from the Swedish federal government under the ALF (agreement on medical training and research).

\section{References}

1 Rosen T \& Bengtsson BA. Premature mortality due to cardiovascular disease in hypopituitarism. Lancet 1990336 285-288.

(doi:10.1016/0140-6736(90)91812-O)

2 Tomlinson JW, Holden N, Hills RK, Wheatley K, Clayton RN, Bates AS, Sheppard MC \& Stewart PM. Association between premature mortality and hypopituitarism. West Midlands Prospective Hypopituitary Study Group. Lancet 2001357 425-431. (doi:10.1016/ S0140-6736(00)04006-X)

3 Pappachan JM, Raskauskiene D, Kutty VR \& Clayton RN. Excess mortality associated with hypopituitarism in adults: a meta-analysis of observational studies. Journal of Clinical Endocrinology and Metabolism 2015100 1405-1411. (doi:10.1210/jc.2014-3787)

4 Nilsson B, Gustavasson-Kadaka E, Bengtsson BA \& Jonsson B. Pituitary adenomas in Sweden between 1958 and 1991: incidence, survival, and mortality. Journal of Clinical Endocrinology and Metabolism 200085 1420-1425. (doi:10.1210/jcem.85.4.6498)

5 Sherlock M, Ayuk J, Tomlinson JW, Toogood AA, Aragon-Alonso A, Sheppard MC, Bates AS \& Stewart PM. Mortality in patients with pituitary disease. Endocrine Reviews 201031 301-342. (doi:10.1210/ er.2009-0033)

6 Olsson DS, Nilsson AG, Bryngelsson IL, Trimpou P, Johannsson G $\&$ Andersson E. Excess mortality in women and young adults with nonfunctioning pituitary adenoma: a Swedish nationwide study. Journal of Clinical Endocrinology and Metabolism 2015100 2651-2658. (doi:10.1210/jc.2015-1475)

7 Gaillard RC, Mattsson AF, Akerblad AC, Bengtsson BA, Cara J, FeldtRasmussen U, Koltowska-Haggstrom M, Monson JP, Saller B, Wilton P et al. Overall and cause-specific mortality in GH-deficient adults on GH replacement. European Journal of Endocrinology 2012166 1069-1077. (doi:10.1530/EJE-11-1028)

8 Sherlock M, Reulen RC, Alonso AA, Ayuk J, Clayton RN, Sheppard MC, Hawkins MM, Bates AS \& Stewart PM. ACTH deficiency, higher doses of hydrocortisone replacement, and radiotherapy are independent predictors of mortality in patients with acromegaly. Journal of Clinical Endocrinology and Metabolism 200994 4216-4223. (doi:10.1210/jc.2009-1097)

9 Zueger T, Kirchner P, Herren C, Fischli S, Zwahlen M, Christ E \& Stettler C. Glucocorticoid replacement and mortality in patients with nonfunctioning pituitary adenoma. Journal of Clinical Endocrinology and Metabolism 201297 1938-1942. (doi:10.1210/jc.2012-2432)

10 Ludvigsson JF, Andersson E, Ekbom A, Feychting M, Kim JL, Reuterwall C, Heurgren M \& Olausson PO. External review and validation of the Swedish national inpatient register. BMC Public Health 201111 450. (doi:10.1186/1471-2458-11-450)

11 Nielsen EH, Lindholm J \& Laurberg P. Use of combined search criteria improved validity of rare disease (craniopharyngioma) diagnosis in a national registry. Journal of Clinical Epidemiology 201164 1118-1126.
12 Olsson DS, Andersson E, Bryngelsson IL, Nilsson AG \& Johannsson G. Excess mortality and morbidity in patients with craniopharyngioma, especially in patients with childhood onset: a population-based study in sweden. Journal of Clinical Endocrinology and Metabolism 2015100 467-474. (doi:10.1210/jc.2014-3525)

13 Altman DG \& Bland JM. Interaction revisited: the difference between two estimates. BMJ 2003326 219. (doi:10.1136/bmj.326.7382.219)

14 Nielsen EH, Lindholm J \& Laurberg P. Excess mortality in women with pituitary disease: a meta-analysis. Clinical Endocrinology 200767 693-697. (doi:10.1111/cen.2007.67.issue-5)

15 Ragnarsson O, Nystrom HF \& Johannsson G. Glucocorticoid replacement therapy is independently associated with reduced bone mineral density in women with hypopituitarism. Clinical Endocrinology 201276 246-252. (doi:10.1111/j.1365-2265.2011.04174.x)

16 Bulow B, Hagmar L, Eskilsson J \& Erfurth EM. Hypopituitary females have a high incidence of cardiovascular morbidity and an increased prevalence of cardiovascular risk factors. Journal of Clinical Endocrinology and Metabolism 200085 574-584. (doi:10.1210/jcem.85.2.6346)

17 Nielsen EH, Lindholm J, Laurberg P, Bjerre P, Christiansen JS, Hagen C, Juul S, Jorgensen J, Kruse A \& Stochholm K. Nonfunctioning pituitary adenoma: incidence, causes of death and quality of life in relation to pituitary function. Pituitary 2007 10 67-73. (doi:10.1007/ s11102-007-0018-x)

18 Holmer H, Svensson J, Rylander L, Johannsson G, Rosen T, Bengtsson BA, Thoren M, Hoybye C, Degerblad M, Bramnert M et al. Nonfatal stroke, cardiac disease, and diabetes mellitus in hypopituitary patients on hormone replacement including growth hormone. Journal of Clinical Endocrinology and Metabolism 200792 3560-3567. (doi:10.1210/jc.2007-0458)

19 Attanasio AF, Mo D, Erfurth EM, Tan M, Ho KY, Kleinberg D, Zimmermann AG, Chanson P \& International Hypopituitary Control Complications Study Advisory Board. Prevalence of metabolic syndrome in adult hypopituitary growth hormone (GH)-deficient patients before and after GH replacement. Journal of Clinical Endocrinology and Metabolism 201095 74-81. (doi:10.1210/jc.2009-1326)

20 Filipsson H, Monson JP, Koltowska-Haggstrom M, Mattsson A \& Johannsson G. The impact of glucocorticoid replacement regimens on metabolic outcome and comorbidity in hypopituitary patients. Journal of Clinical Endocrinology and Metabolism 200691 3954-3961. (doi:10.1210/jc.2006-0524)

21 Burman P, Mattsson AF, Johannsson G, Hoybye C, Holmer H, Dahlqvist P, Berinder K, Engstrom BE, Ekman B, Erfurth EM et al. Deaths among adult patients with hypopituitarism: hypocortisolism during acute stress, and de novo malignant brain tumors contribute to an increased mortality. Journal of Clinical Endocrinology and Metabolism 201398 1466-1475. (doi:10.1210/jc.2012-4059)

22 Wuster C, Abs R, Bengtsson BA, Bennmarker H, FeldtRasmussen U, Hernberg-Stahl E, Monson JP, Westberg B, Wilton P, Group KS et al. The influence of growth hormone deficiency, growth hormone replacement therapy, and other aspects of hypopituitarism on fracture rate and bone mineral density. Journal of Bone and Mineral Research 200116 398-405. (doi:10.1359/ jbmr.2001.16.2.398)

23 Holmes SJ, Economou G, Whitehouse RW, Adams JE \& Shalet SM. Reduced bone mineral density in patients with adult onset growth hormone deficiency. Journal of Clinical Endocrinology and Metabolism 199478 669-674. (doi:10.1210/jcem.78.3.8126140)

24 Rosen T, Wilhelmsen L, Landin-Wilhelmsen K, Lappas G \& Bengtsson BA. Increased fracture frequency in adult patients with hypopituitarism and GH deficiency. European Journal of Endocrinology 1997137 240-245. (doi:10.1530/eje.0.1370240)

Received 24 February 2016

Revised version received 30 April 2016

Accepted 4 May 2016 